\title{
Microscopic calculations of $\Lambda$ single-particle energies
}

\begin{abstract}
A binding energy data for total baryon number A Ò208 and for ₹ angular momenta ǎs Ò3 are analyzed in terms of phenomenological (but generally consistent with meson-exchange) § $\mathrm{N}$ and $\$ \mathrm{NN}$ potentials. The Fermi hypernetted chain technique is used to calculate the expectation values for the $\$$ binding to nuclear matter. Accurate effective $\$ \mathrm{~N}$ and $\$ \mathrm{NN}$ potentials are obtained which are folded with the core-nucleus nucleon densities to calculate the $\$$ single-particle potential Us ( $r$ ). We use a dispersive ANN potential but also include an explicit $f$ dependence to allow for reduced repulsion in the surface, and the best fits have a large $f$ dependence giving consistency with the variational Monte Carlo calculations for 5 \$ He. The exchange fraction of the $\$ \mathrm{~N}$ space-exchange potential is found to be $0.2-0.3$ corresponding to $\mathrm{m} * \boldsymbol{\varsigma} \simeq(0.74-0.82) \mathrm{m}$. Charge-symmetry breaking is found to be significant for heavy hypernuclei with a large neutron excess, with a strength consistent with that obtained from the $\mathrm{A}=4$ hypernuclei.
\end{abstract}

Keyword: \$ single-particle energies; Microscopic calculations 